\title{
An Evolving Role for Cancer Rehabilitation in the Era of Low-Dose Lung Computed Tomography Screening
}

\author{
Sean Robinson Smith, MD, Ashish Khanna, MD, Eric M. Wisotzky, MD
}

\begin{abstract}
Lung cancer is the number one cause of cancer-related death worldwide, and is often detected in the later stages. Use of lowdose chest computed tomography in at-risk patients provides earlier detection and is being adopted as the standard screening tool, replacing less precise methods of radiography and sputum cytology. In the past, late detection of disease meant that rehabilitation interventions attempted to salvage function and to improve aerobic capacity to the point where patients could tolerate the sometimes-extensive oncologic treatment, including lobectomy or pneumonectomy. Earlier detection may shift this toward more often addressing specific neuromusculoskeletal impairments, such as postthoracotomy pain or peripheral neuropathy, as patients with early-stage disease may not be as debilitated by chronic disease or metastases as those with late-stage lung cancer. Patients with advanced disease, however, will still require rehabilitation interventions, and this fragile population creates unique challenges. Rehabilitation professionals should look for ways to expand care to lung cancer patients, as both the number of those treated and the 5-year survival rate are expected to increase.
\end{abstract}

\section{Introduction}

Lung cancer is one of the most common cancer diagnoses worldwide, and remains the number one cause of cancer-related death in both men and women $[1,2]$. During 2016, the incidence of new cases in the United States was estimated to be 224,400 , representing about $14 \%$ of all cancer diagnoses [3]. Approximately 415,000 Americans have a diagnosis of lung cancer, 82\% of whom are age 60 years or older, with the average age at diagnosis at 70 (less than 2\% are younger than 45 years) [4]. Smoking remains the primary risk factor for developing disease, and nonsmokers who are exposed to second-hand smoke by living with a smoker have a $20 \%$ $30 \%$ increased risk of developing lung cancer over that of the general population. About $10 \%$ of cases arise from toxic exposure, such as to radon or asbestos [3]. The risk of developing lung cancer without any of these factors is unclear. Of note, the majority of people with a history of lung cancer have had their diagnoses made within the past 5 years.

Primary lung carcinoma is a heterogeneous group of tumor subtypes, with the most important distinction being between small-cell and non-small-cell lung cancer (NSCLC). Treatment of NSCLC often involves a combination of surgery, chemotherapy, and/or radiation, whereas small-cell lung cancer often does not involve surgery unless there are focal, large areas of tumor burden in a location amenable to resection [5]. In those with early-stage lung cancer, surgical resection of the tumor is typically the first aspect of oncologic management [6].

The diagnosis of lung cancer is, unfortunately, often late, as patients may be asymptomatic with early-stage lung cancer, and up to two-thirds of patients have metastases at the time of diagnosis [7]. Furthermore, the stage at diagnosis is closely associated with survival, with a 5-year survival of only $6 \%$ for patients with metastases present on initial diagnosis, compared with $85 \%$ for patients with stage IA disease [8]. Only about $15 \%$ of patients are diagnosed with stage I disease $[9,10]$.

Recommendations for lung screening vary by organization, but most generally agree that testing should be administered in individuals 55-74 years of age with a smoking history of $\geq 30$ pack-years (defined as "heavy smoking") and who either continue to smoke or have quit within the past 15 years. Additional recommendations include screening patients with a smoking history 
of $\geq 20$ pack-years who have additional risk factors, such as environmental exposure to hazardous chemicals (other than second-hand smoke) [11]. Screening has generally consisted of radiography and/or sputum cytology, with computed tomography (CT) reserved for patients with suspicious but inconclusive findings on these modalities [12].

Due to technological advances and the low rate of early disease detection, in 2014 the US Preventative Services Task Force recommended annual screening for lung cancer with low-dose computed tomography (LDCT) in adults 55 to 80 years of age who have a smoking history of 30 pack-years and currently smoke or have quit within the past 15 years [13]. LDCT uses approximately $20 \%$ less radiation than traditional CT screening, making this protocol safer for patients who may need serial imaging [14]. These guidelines have resulted in Medicare coverage of LDCT for patients meeting the aforementioned criteria.

The largest trial looking at the benefits of lung screening, the National Lung Screening Trial (NLST), showed a reduction in lung cancer mortality of $16 \%$ and a reduction in all-cause mortality of $6.7 \%$ [14]. The use of alternative and more aggressive screening criteria using LDCT are being investigated, potentially preventing another 16,000 additional deaths because of improved identification of at-risk patients $[15,16]$. Multiple studies continue to evaluate the efficacy of LDCT screening in an effort to improve specificity, to improve detection, to save costs, and reduce mortality [9].

Earlier detection through LDCT has the potential to increase the cure rate of lung cancer through earlier detection and to reduce treatment-associated morbidity due to a smaller tumor burden at the time of diagnosis [9]. It also may change the approach to rehabilitation of lung cancer patients. Rehabilitation interventions often help to optimize a patient for oncologic treatment including surgery, and to manage symptoms such as pain and weakness. With earlier detection, rehabilitation programs may have more opportunities to prevent and to manage symptoms and functional deficits throughout a longer timeframe, and perhaps more often into long-term survivorship.

\section{Symptoms and Rehabilitation Needs of Lung Cancer Patients}

Lung cancer and its treatment have the potential to cause significant symptom burden due to worsening pain, dyspnea, fatigue, cognitive deficits, impaired balance, depression, and other symptoms that contribute to a decrease in health-related quality of life (HRQoL) $[17,18]$. When combined with potential debility from chronic illness such as chronic obstructive pulmonary disease (COPD), diabetes, and peripheral vascular disease, these symptoms can have a negative impact on function and social role [19]. Physical activity and quality of life are lower in patients with NSCLC compared to healthy controls [20], and low physical activity [21] and HRQoL [22] are negative prognostic factors for survival. In advanced stages, metastases to bones [23] and the central nervous system [24] may cause functional decline due to neurologic deficits and pain, necessitating rehabilitation intervention to reduce symptom burden.

The surgical treatment for lung cancer can be deleterious to patients' function, with surgery typically consisting of lobectomy or pneumonectomy for NSCLC patients with locoregional disease. Although both modalities lead to increased dyspnea and short-term worsening of pain and physical function, patients who undergo lobectomy have a reduced burden and duration of symptoms compared to pneumonectomy patients. Those undergoing pneumonectomy may never return to preoperative baseline in physical function, and also frequently experience dyspnea and/or shoulder pain $[25,26]$. In addition, exercise capacity is decreased in lung cancer patients in general and further by surgery [21]. The larger the resected disease, the more impact that surgery appears to have on HRQoL, and more than $20 \%$ of patients report having 3 or more severe symptoms after surgery $[27,28]$. This suggests that earlier detection of smaller disease with LDCT may reduce the negative impact of surgery.

Extensive disease and/or premorbid chronic illness may also increase the risk of negative outcomes after surgery, including prolonged hospital stays and unanticipated readmissions. In a survey of 11,500 patients undergoing tumor resection, $\mathrm{Hu}$ et al found that approximately $13 \%$ of lung cancer patients were readmitted to the hospital within 90 days for complications from surgery. Of those who were readmitted, there was a 6-fold increase in 90-day mortality. The most common readmitting diagnoses were respiratory insufficiency, pneumonia, pneumothorax, and cardiac complications [29]. This again highlights the potential benefits of early diagnosis, as chronic comorbidities may be less severe in patients with early-stage disease.

Chemotherapy, which often follows surgery and consists of platinum-containing antineoplastic agents, can contribute to peripheral neuropathy, causing pain and gait abnormalities as well as cognitive deficits. Patients who receive chemotherapy have worse HRQoL at 3-months postoperatively compared to those without [30], and symptoms of neuropathy often did not abate over 2.5 years of follow-up in one longitudinal study [31].

Furthermore, radiation also has potentially negative effects, with pneumonitis and dyspnea being the most common short- and long-term complication, and extensive fibrosis of the lung parenchyma and surrounding neuromusculoskeletal structures being a possible late effect [32]. It is unclear whether the incidence of radiation fibrosis would decrease with 
the earlier detection of disease and potentially increased use of stereotactic body radiation therapy, a more precise method of administering radiation that can be used for smaller tumors and that uses less total radiation dosing. In the setting of brain metastases, whole-brain radiation therapy is also a predictor for development of impaired mobility [24]. Of note, patients receiving chemotherapy and/or radiation therapy self-report less physical activity compared to long-term lung cancer survivors and those who have recently undergone surgery [33]. It is unclear whether earlier detection of disease through the use of LDCT would obviate the need for chemoradiation therapy.

Pain is perhaps the most distressing symptom in lung cancer patients, with up to $92 \%$ of survivors $1-6$ years postoperative reporting some level of pain [34]. One source of pain is post-thoracotomy pain, which is thought to be due to injury of the intercostal nerve(s) during surgery, may be acute or chronic, and is often neuropathic in nature. Post-thoracotomy pain may be the most common postoperative source of pain [35], and it affects approximately $50 \%$ of lung cancer patients who have lung surgery, with about $5 \%$ of patients developing chronic, severe, and disabling pain [36]. Rehabilitation interventions aim to reduce neuropathic pain, to preserve pulmonary hygiene during periods of severe pain that prevent deep breathing, and to progressively improve mobility in patients whose function is compromised by the pain.

\section{Rehabilitation Interventions for Lung Cancer: A Changing Role With Earlier Detection}

Rehabilitation in lung cancer may be performed at any time along the continuum of care, and traditionally involves strengthening muscles of respiration, aerobic conditioning, strategies for energy conservation, as well as management of pain and other symptoms. Rehabilitation before oncologic care, often referred to as prehabilitation, may also be used to optimize patient function before treatment or to improve patients' respiratory and/or performance status to the point at which they are candidates for surgery or chemotherapy. To our knowledge, prehabilitation has never been directly compared to postsurgical rehabilitation in lung cancer patients, but either approach may confer a benefit to the patient, depending on the stage and type of disease and on the patient's premorbid physical health.

Numerous studies have found benefits in rehabilitation of lung cancer patients, particularly improved walking distance and patient-reported symptoms in early-stage lung cancer, but relatively few studies have looked at exercise and rehabilitation in advanced lung cancer [37]. This is despite the fact that patients with advanced lung cancer have significant functional impairment and musculoskeletal comorbidities and often desire rehabilitation services [38]. In fact, patients have been shown to prefer palliation of symptoms and rehabilitation to improve HRQoL even if it means a trade-off with survival [39], and HRQoL is directly linked to symptom burden [40] and physical function [41] in lung cancer patients. Although lung cancer patients and their families are generally supportive of rehabilitation interventions, they may not be comfortable performing a self-directed program. This is true particularly in patients with advanced disease, suggesting that a structured rehabilitation program is needed [42].

Postsurgical pulmonary rehabilitation has been shown to improve various measures of pulmonary and physical function, particularly when a high-intensity aerobic conditioning approach is tolerated by patients [43]. Edvardsen et al found that a 20 -week intensive regimen starting 5-7 weeks after lung cancer resection improved exercise tolerance, functional fitness, muscle mass, and strength. For patients with early-stage tumors receiving curative surgery, postoperative rehabilitation helps to improve both pulmonary and gross physical function [44] and to reduce distress [45], and should be considered. Other prospective trials without a control arm suggest a benefit of postoperative rehabilitation improving physical function in patients with more advanced disease [46-48].

Unfortunately, rehabilitation after surgery or chemotherapy is not always successful in improving physical function or quality of life. This is in part due to the myriad comorbidities in lung cancer patients, including COPD and musculoskeletal pain. For example, Stigt et al found that, in a randomized controlled trial of patients with advanced lung cancer undergoing rehabilitation consisting of exercise, pain management, and social work support, Six-Minute Walk Test (6MWT) distance was increased by $35 \mathrm{~m}$ compared to that of controls, but pain was worse and quality of life was no better [49]. This corroborates findings from a similar study that did not provide pain management or psychosocial support, and that did not even find improvement in the 6MWT results compared to those in controls [50]. Another study found that postoperative pulmonary rehabilitation alone does improve physical activity, but not to the patients' preoperative baseline [51]. These findings suggest that when disease is detected late, postoperative rehabilitation interventions may be less effective. With earlier detection through the use of LDCT, rehabilitation interventions may be more effective and therefore presumably may be used more.

Finally, rehabilitation interventions are also needed for specific impairments, such as post-thoracotomy pain, for which pharmacologic [52,53] and procedural interventions $[54,55]$ administered by a physiatrist may reduce symptom burden. Other musculoskeletal conditions associated with lung cancer, including management of the malignant spine, would benefit from physiatric management [56]. 


\section{Prehabilitation as a Way to Prepare Patients for Oncologic Treatment and to Improve Outcomes}

Prehabilitation, a multimodal intervention to prepare a patient for upcoming treatment, is an important intervention to improve physical function and to reduce symptom burden after surgery, and may be more effective than postoperative rehabilitation alone. A recent subject matter expert panel led by Carli and Silver concluded that in addition to aerobic exercise, prehabilitation before lung cancer surgery should consist of nutrition optimization with an emphasis on protein supplementation and glycemic control, as well as a smoking cessation program to optimize surgical outcomes [57]. A recent meta-analysis by Garcia et al of prehabilitation in lung cancer patients before surgery found that there appears to be a benefit in pulmonary function as measured by forced vital capacity (FVC) and forced expiratory volume in 1 second (FEV1), as well as in reducing postoperative complications and length of stay [58]. Given the heterogeneity of outcome measures as it related to HRQoL, gross physical function, and exercise capacity, this analysis was unable to assess the impact of prehabilitation on HRQoL.

In reviewing individual studies that the meta-analysis by Garcia et al evaluated, however, it is apparent that prehabilitation does improve both physical function and $\mathrm{HRQ}$ oL in lung cancer patients, albeit with inconsistent and varying outcome measures that render meta-analysis inconclusive [44,59-61]. For example, one randomized controlled study assessed lung cancer patients who underwent 4 weeks of preoperative pulmonary rehabilitation consisting of breathing and aerobic exercise compared to patients receiving routine chest physical therapy, and found that the prehabilitation group had improved 6MWT distance $(P<.05)$, FVC $(P<.05)$, maximal inspiratory and expiratory pressure $(P<.05)$, lower postoperative morbidity $(P=.01)$, length of stay $(P=.04)$, and days with chest tubes in place $(P=.03)$ [62]. This study specifically looked at patients with premorbid pulmonary comorbidities such as COPD, showing that aggressive prehabilitation is able to improve function even in lung cancer patients with chronic illness.

A similar controlled study looked at NSCLC patients with moderate-to-severe COPD receiving prehabilitation before surgery compared to no intervention, and also found that the intervention group had fewer chest tube days $(P=.04)$ and that the decline in length of stay postoperatively approached statistical significance $(P=.058)[63]$.

Finally, and perhaps most significant, lung cancer patients whose pulmonary function was so poor secondary to COPD such that surgery was too high risk and who underwent pulmonary prehabilitation showed improvement to the point where they were able to tolerate and eventually to undergo surgical resection of stage I and stage || tumors [64], demonstrating the potentially lifesaving role of prehabilitation. This underscores the value of prehabilitation in patients with premorbid chronic disease, and how this may be an essential component of care, particularly given that postoperative rehabilitation alone may not be adequate in improving function. With the advent of LDCT, there will likely be more patients whose cancer is diagnosed in earlier stages, thus increasing the need for prehabilitation services to prepare these patients for surgery. Before LDCT, cancer diagnoses in these patients may have been made at later stages, and therefore surgery would not have been an option and prehabilitation not indicated. By diagnosing these patients with cancer earlier through LDCT, prehabilitation has the potential to be lifesaving and should be considered as an essential component of lung cancer oncologic treatment, improving access to surgery and potentially survival outcomes.

In addition to lowering postoperative complications, prehabilitation may reduce postoperative length of stay. Weinstein et al found that, in lung cancer patients, exercise capacity as measured by treadmill-based physical activity was inversely related to postoperative length of stay, even after controlling for method of surgery, gender, presence of dyspnea, and smoking history $(P<.003)$ [65]. When exercise capacity was poor, length of stay nearly doubled. This is of particular importance, not just in reducing morbidity, but in lowering health care costs and improving health care efficiency, given the changing health care landscape to reward care quality and use of bundled payment models. Prehabilitation may prove to be a cost-effective rehabilitation intervention, and LDCT may allow earlier intervention and potentially less morbidity and more cost savings, an increasingly important aspect of care as insurance payors switch to quality-based care models.

For patients without chronic disease such as COPD, undergoing prehabilitation before tumor resection may be challenging because of the importance of beginning oncologic treatment as soon as possible. Nevertheless, a randomized controlled study comparing usual care to patients undergoing just a 1-week prehabilitation course consisting of respiratory muscle training, cardiovascular exercise, education, and bronchodilators (as needed) found that prehabilitation led to improved dyspnea, Thoracoscore (a measure used to predict postoperative mortality and complications), and performance status. Compared to usual care, patients who went through 1 week of prehabilitation had fewer postoperative complications $(37.5 \%$ to $5.3 \%, P<.015)$ and no mortality (compared to $11.1 \%$ in the control arm, $P=.05$ ) [66]. If even a 1 -week course of prehabilitation is untenable, pulmonary rehabilitation postoperatively but during adjuvant radiation and chemotherapy was shown to improve FVC and FEV1 $(P=.0096$ and $P<.0001$, respectively), but the benefit was lower in 
patients with poorer pulmonary status, suggesting that rehabilitation interventions would be even more impactful were these patients to undergo prehabilitation for a short time before surgery [67]. Tokarski et al corroborated findings from this study by observing that rehabilitation during adjuvant chemotherapy improved pulmonary function $(P<0.05)$ [68].

Although helpful for patients with comorbidities such as COPD, the utility of prehabilitation interventions for early-stage tumors is not entirely clear. For example, not all stage I lung cancer patients need to undergo surgery and chemoradiation therapy immediately upon detection, creating uncertainty as to when and how a rehabilitation program is best implemented. Progression of nonsolid, malignant ground glass nodules is rare, with one study finding a $100 \%$ survival rate of patients with these ground glass nodules at 6.5 years [69]. Although this may open an opportunity for prehabilitation as patients diagnosed with early disease are expected to increase in number with LDCT, these patients may not undergo surgery for some time and may not have significant comorbidities necessitating intervention. In addition, disease may also be monitored for some time, as one study found that LDCT screening was associated with a $23 \%$ false-positive rate for even advanced stage disease, meaning that although LDCT screening is being implemented and improved upon, treatment may be on hold for some patients whose diagnosis is unclear and who are undergoing active surveillance [70]. Nevertheless, programs to optimize fitness and function in these patients may reduce morbidity, should disease ever progress and/or treatment be needed, and patients will need to undergo active surveillance to ensure that they are not declining functionally or developing pain.

\section{Survivorship}

Five-year survival rates have increased in NSCLC patients from $11.4 \%$ in 1975 to $17.3 \%$ in 2009 , with rates increasing to nearly $40 \%$ in patients with localized disease $[71,72]$. The use of LDCT should improve survival through earlier detection, potentially creating a large population of patients with survivorship needs in a cohort that previously had lower survival rates compared to patients with other tumor types.

Lung cancer survivors experience lasting effects of both the disease and treatment, with lower HRQoL found in $35 \%$ of patients 5 years after completion of treatment [73], and $60 \%$ of long-term survivors reporting at least one chronic, symptomatic comorbidity [74]. Compared to controls who were never diagnosed with lung cancer, long-term survivors report lower physical health, even if their cancer was detected early [75] and two-thirds of long-term NSCLC survivors do not meet national physical activity recommendations [76]. The most common physical symptoms experienced by long-term NSCLC survivors include impaired respiration, fatigue, neuropathic pain, and post-thoracotomy pain [17].

As described previously, exercise-based rehabilitation interventions may improve physical function and reduce symptoms related to dyspnea and fatigue. In long-term survivors, however, the benefits of these interventions are less clear. Yang et al found that exercise improved HRQoL in 5-year NSCLC survivors, despite the fact that symptom burden was not reduced [70]. In addition, self-reported physical activity in lung cancer survivors improves physical, emotional, and mental well-being, although it has no impact on disease recurrence [33].

Furthermore, impairment-driven rehabilitation that addresses specific problems in long-term lung cancer survivors-such as chronic post-thoracotomy pain, shoulder pain, and peripheral neuropathy-may be more important in improving function and reducing distress than general exercise programs, which appear to improve HRQoL but not reduce symptoms [77]. With the use of LDCT screening, patients' premorbid health may be better than in patients whose diagnoses were made in previous years, given earlier disease detection, allowing physiatrists and other members of the cancer rehabilitation team to focus on particular problems in patients with relatively preserved general physical function and exercise capacity.

Finally, long-term survivors also experience considerable psychosocial distress, including depression and anxiety related to their disease, and subsequent decline in function, with at least $40 \%$ of NSCLC survivors expressing distress [78]. A survivor's social role is often affected, with lung cancer patients having the highest rate of leaving the workplace of all cancer patients $[79,80]$. This chronic symptom burden, along with the increased focus on management of survivorship needs including rehabilitation needs [81,82], creates an expanded role for rehabilitation interventions to restore function and to address needs over a potentially longer period of time than before LDCT.

In addition to psychosocial support services and interventions by trained rehabilitation psychologists to address the impact of reduced function on survivors' emotional well-being, walking and exercise programs also have the potential to reduce distress. One randomized controlled trial of a home-based walking program found that survivors still symptomatic from lung cancer and/or its treatment had reduced anxiety and depression compared to controls [83]. Symptoms of severe anxiety may increase perceived dyspnea, so techniques to manage anxiety may reduce the effects of dyspnea [84]. The impact of LDCT on survivors' emotional health remains to be seen; however, with presumably more long-term survivors, these problems will likely become more prevalent and will open up opportunities for physiatrists to provide multiple 
supportive care services, including those of rehabilitation psychology providers.

\section{Conclusion}

The use of LDCT for lung screening presents an opportunity for increased rehabilitation intervention to restore function, although the scope of this opportunity, and specifics as to how rehabilitation would best be carried out, remain to be seen. With earlier detection of lung cancer, patients may have less debility from chronic disease at the time of diagnosis and throughout the course of treatment, although problems such as post-thoracotomy pain and chemotherapy-induced peripheral neuropathy are unlikely to be decreased. In fact, more total patients may be diagnosed with lung cancer, and therefore the number of patients in need of rehabilitation services may increase. Postoperative rehabilitation programs may be more effective in the era of LDCT and earlier detection because of reduced severity of chronic comorbidities, pain, and the need for less extensive surgery. Although rehabilitation care for lung cancer patients will doubtless change with the advent of LDCT screening, a multimodal approach will likely improve functional outcomes and possibly survival if more patients are able to undergo surgery or have less morbidity after surgery through prehabilitation and rehabilitation.

With LDCT potentially resulting in more diagnoses at cancer stages I and II, prehabilitation interventions may play a significantly increased role, given that it has the potential to prepare these patients for surgery and to improve outcomes, including survival and the number of patients who become suitable surgical candidates with a prehabilitation regimen. What is unclear is whether earlier detection would mean that patients would be less impaired by deconditioning related to chronic disease. Modifications to preoperative conditioning regimens may need to be made, such as shortening the duration of a program, as disease detected earlier may not require prehabilitation simply to prepare patients for surgery if the cancer or underlying chronic conditions such as COPD are not as advanced. More research is needed to determine the optimal degree of rehabilitation intervention, as short- and long-term prehabilitation programs have not been directly compared, nor have prehabilitation and postoperative rehabilitation programs.

Given the success of even short-term prehabilitation programs, and the significant impact of poor patient exercise capacity on postoperative hospital length of stay, rehabilitation providers must evaluate interventions in the context of cost savings; if a program reduces health care costs, it is much more likely to be adopted by all insurance payors. Prehabilitation in particular has the potential to significantly support value-based care by reducing cost, improving surgical outcomes, and improving patient satisfaction and HRQoL.

The subspecialty of cancer rehabilitation is experiencing rapid growth, due to both an increased number of cancer survivors and renewed interest on behalf of physiatry. It is imperative that physiatrists and other members of the rehabilitation team to stay up-to-date on oncology protocols such as the use of LDCT, and explore opportunities to expand beneficial rehabilitation services. Program development to accommodate an increased number of patients, and to efficiently manage these patients to reduce health care costs, represents an opportunity to improve patient care, expand the field, and research new avenues of intervention.

\section{References}

1. Travis WD, Brambilla E, Noguchi M, et al. International Association for the Study of Lung Cancer/American Thoracic Society/European Respiratory Society International Multidisciplinary Classification of Lung Adenocarcinoma. J Thorac Oncol 2011;6:244-285.

2. World Health Organization. Cancer: Fact sheet no. 297. 2015. Geneva: World Health Organization; 2015.

3. American Lung Association. Lung Cancer Fact Sheet. Edited 3 Nov 2016.

4. Siegel RL, Miller KD, Jemal A. Cancer statistics, 2016. CA Cancer J Clin 2016;66:7-30.

5. Molina JR, Yang P, Cassivi SD, Schild SE, Adjei AA. Non-small cell lung cancer: Epidemiology, risk factors, treatment, and survivorship. Mayo Clin Proc 2008;83:584-594.

6. Detterbeck FC, Boffa DJ, Tanoue LT. The new lung cancer staging system. Chest J 2009;136:260-271.

7. Van Klaveren RJ. Lung cancer screening. Eur J Cancer 2011; 47(Suppl 3):S147-S155.

8. Goldstraw P, Chansky K, Crowley J, et al. The IASLC Lung Cancer Staging Project: Proposals for revision of the TNM stage groupings in the forthcoming (eighth) edition of the TNM classification for lung cancer. J Thorac Oncol 2016;11:39-51.

9. Heuvelmans MA, Groen HJ, Oudkerk M. Early lung cancer detection by low-dose CT screening: Therapeutic implications. Expert Rev Respir Med 2017;11:89-100.

10. Birim Ö, Kappetein AP, Takkenberg JJ, et al. Survival after pathological stage IA nonsmall cell lung cancer: Tumor size matters. Ann Thorac Surg 2005;79:1137-1141.

11. Smith RA, Andrews K, Brooks D, et al. Cancer screening in the United States, 2016: A review of current American Cancer Society guidelines and current issues in cancer screening. CA Cancer J Clin 2016;66:96-114.

12. Midthun DE. Early detection of lung cancer. Cancer Prev Res 2016;5.

13. Moyer VA. Screening for lung cancer: US Preventive Services Task Force recommendation statement. Ann Intern Med 2014;160: 330-338.

14. National Lung Screening Trial Research Team. Reduced lungcancer mortality with low-dose computed tomographic screening. N Engl J Med 2011;365:395-409.

15. Gould MK. Who should be screened for lung cancer? And who gets to decide? JAMA 2016;315:2279-2281.

16. Katki HA, Kovalchik SA, Berg CD, et al. Development and validation of risk models to select ever-smokers for $\mathrm{CT}$ lung cancer screening. JAMA 2016;315:2300-2311.

17. Hung R, Krebs P, Coups EJ, et al. Fatigue and functional impairment in early-stage non-small cell lung cancer survivors. J Pain Symptom Manage 2011;41:426-435. 
18. Shannon VR. Role of pulmonary rehabilitation in the management of patients with lung cancer. Curr Opin Pulm Med 2010;16:334-339.

19. Polanski J, Jankowska-Polanska B, Rosinczuk J, et al. Quality of life of patients with lung cancer. OncoTargets Ther 2016;9:1023.

20. Granger CL, Denehy L, McDonald CF, Irving L, Clark RA. Physical activity measured using global positioning system tracking in non-small cell lung cancer: An observational study. Integr Cancer Ther 2014;13:482-492.

21. Jones LW, Peddle CJ, Eves ND, et al. Effects of presurgical exercise training on cardiorespiratory fitness among patients undergoing thoracic surgery for malignant lung lesions. CA Cancer J Clin 2007; 110:590-598.

22. Schild SE, Tan AD, Wampfler JA, et al. A new scoring system for predicting survival in patients with non-small cell lung cancer. Cancer Med 2015;4:1334-1343.

23. Cheville AL, Murthy NS, Basford JR, et al. Imaging and clinical characteristics predict near-term disablement from bone metastases: Implications for rehabilitation. Arch Phys Med Rehabil 2016; 97:53-60.

24. Cheville AL, Basford JR, Parney I, et al. A nested cohort study to identify characteristics that predict near-term disablement from lung cancer brain metastases. Arch Phys Med Rehabil 2016; 98:303-311.

25. Balduyck B, Hendriks J, Lauwers P, et al. Quality of life evolution after lung cancer surgery: A prospective study in 100 patients. Lung Cancer 2007;56:423-431.

26. Handy JR, Asaph JW, Skokan L, et al. What happens to patients undergoing lung cancer surgery? Outcomes and quality of life before and after surgery. Chest J 2002;122:21-30.

27. Schulte T, Schniewind B, Dohrmann P, et al. The extent of lung parenchyma resection significantly impacts long-term quality of life in patients with non-small cell lung cancer. Chest J 2009;135: 322-329.

28. Lowery AE, Krebs P, Coups EJ, et al. Impact of symptom burden in post-surgical non-small cell lung cancer survivors. Support Care Cancer 2014;22:173-180.

29. Hu Y, McMurry TL, Isbell JM, et al. Readmission after lung cancer resection is associated with a 6-fold increase in 90-day postoperative mortality. J Thorac Cardiovasc Surg 2014;148:2261-2267.

30. Winton T, Livingston R, Johnson D, et al. Vinorelbine plus cisplatin vs. observation in resected non-small-cell lung cancer. N Engl J Med 2005;352:2589-2597.

31. Bezjak A, Lee CW, Ding K, et al. Quality-of-life outcomes for adjuvant chemotherapy in early-stage non-small-cell lung cancer: Results from a randomized trial. J Clin Oncol 2008;26:5052-5059.

32. Rancati T, Ceresoli GL, Gagliardi G, et al. Factors predicting radiation pneumonitis in lung cancer patients: A retrospective study. Radiother Oncol 2003;67:275-283.

33. Sloan JA, Cheville AL, Liu H, et al. Impact of self-reported physical activity and health promotion behaviors on lung cancer survivorship. Health Qual Life Outcomes 2016;14:1.

34. Iyer S, Roughley A, Rider A, et al. The symptom burden of nonsmall cell lung cancer in the USA: A real-world cross-sectional study. Support Care Cancer 2014;22:181-187.

35. Fortier S, Hanna HA, Bernard A, et al. Comparison between systemic analgesia, continuous wound catheter analgesia and continuous thoracic paravertebral block: A randomised, controlled trial of postthoracotomy pain management. Eur J Anaesthesiol 2012;29:524-530.

36. Rogers ML, Duffy JP. Surgical aspects of chronic post-thoracotomy pain. Eur J Cardio-thoracic Surg 2000;18:711-716.

37. Bade BC, Thomas DD, Scott JB, Silvestri GA. Increasing physical activity and exercise in lung cancer: Reviewing safety, benefits, and application. J Thorac Oncol 2015;10:861-871.

38. Cheville AL, Rhudy L, Basford JR, et al. How receptive are patients with late stage cancer to rehabilitation services and what are the sources of their resistance? Arch Phys Med Rehabil 2016;98: 203-210.
39. Silvestri G, Pritchard R, Welch HG. Preferences for chemotherapy in patients with advanced non-small cell lung cancer: Descriptive study based on scripted interviews. BMJ 1998;317:771-775.

40. Tanaka K, Akechi T, Okuyama T, et al. Impact of dyspnea, pain, and fatigue on daily life activities in ambulatory patients with advanced lung cancer. J Pain Symptom Manage 2002;23:417-423.

41. Brown DJ, McMillan DC, Milroy R. The correlation between fatigue, physical function, the systemic inflammatory response, and psychological distress in patients with advanced lung cancer. CA Cancer J Clin 2005;103:377-382.

42. Rhudy L, Dose AM, Basford J, et al. Caregivers' attitudes toward promoting exercise among patients with late-stage lung cancer. J Commun Support Oncol 2015;3:392-398.

43. Edvardsen E, Skjonsberg OH, Holme I, et al. High-intensity training following lung cancer surgery: A randomised controlled trial. Thorax 2015;70:244-250.

44. Bradley A, Marshall A, Stonehewer L, et al. Pulmonary rehabilitation programme for patients undergoing curative lung cancer surgery. Eur J Cardiothorac Surg 2013;44:e266-e271.

45. Sommer MS, Trier K, Vibe-Petersen J, et al. Changes in healthrelated quality of life during rehabilitation in patients with operable lung cancer: A feasibility study (PROLUCA). Integrat Cancer Ther 2016. E-pub ahead of print.

46. Hwang CL, Yu CJ, Shih JY, et al. Effects of exercise training on exercise capacity in patients with non-small cell lung cancer receiving targeted therapy. Support Care Cancer 2012;20: 3169-3177.

47. Spruit MA, Janssen PP, Willemsen SC, et al. Exercise capacity before and after an 8-week multidisciplinary inpatient rehabilitation program in lung cancer patients: A pilot study. Lung Cancer 2006;52:257-260.

48. Cesario A, Ferri L, Galetta D, et al. Post-operative respiratory rehabilitation after lung resection for non-small cell lung cancer. Lung Cancer 2007; 57:175-180.

49. Stigt JA, Uil SM, van Riesen SJ, et al. A randomized controlled trial of postthoracotomy pulmonary rehabilitation in patients with resectable lung cancer. J Thorac Oncol 2013;8:214-221.

50. Arbane G, Tropman D, Jackson D, Garrod R. Evaluation of an early exercise intervention after thoracotomy for non-small cell lung cancer (NSCLC), effects on quality of life, muscle strength and exercise tolerance: Randomised controlled trial. Lung Cancer 2011;71:229-234.

51. Maeda K, Higashimoto Y, Honda N, et al. Effect of a postoperative outpatient pulmonary rehabilitation program on physical activity in patients who underwent pulmonary resection for lung cancer. Geriatr Gerontol Int 2015;16:550-555.

52. Mishra A, Nar AS, Bawa A, et al. Pregabalin in chronic post-thoracotomy pain. J Clin Diagn Res 2013;7:1659.

53. Yoshimura N, lida H, Takenaka M, et al. Effect of postoperative administration of pregabalin for post-thoracotomy pain: A randomized study. J Cardiothorac Vasc Anesth 2015;29:1567-1572.

54. Saby A, Swaminathan K, Pangarkar S, Tribuzio B. Alleviating thoracotomy pain with intercostal liposomal bupivacaine: A case report. PM R 2016:81119-81122.

55. Biswas S, Verma R, Bhatia VK, et al. Comparison between thoracic epidural block and thoracic paravertebral block for post thoracotomy pain relief. J Clin Diagn Res 2016;10:UC08.

56. Ruppert LM. Malignant spinal cord compression: Adapting conventional rehabilitation approaches. Phys Med Rehabil Clin N Am 2017;28:101-114.

57. Carli F, Silver JK, Feldman LS, et al. Surgical prehabilitation in patients with cancer: State-of-the-science and recommendations for future research from a panel of subject matter experts. Phys Med Rehabil Clin N Am 2017;28:49-64.

58. Garcia RS, Brage MI, Moolhuyzen EG, et al. Functional and postoperative outcomes after preoperative exercise training in patients with lung cancer: A systematic review and meta-analysis. Interact Cardiovasc Thorac Surg 2016;23:486-497. 
59. Granger CL, McDonald CF, Berney S, et al. Exercise intervention to improve exercise capacity and health related quality of life for patients with non-small cell lung cancer: A systematic review. Lung Cancer 2011;72:139-153.

60. Rueda JR, Sola I, Pascual A, et al. Non-invasive interventions for improving well-being and quality of life in patients with lung cancer. Cochrane Database Syst Rev 2011;9:CD004282.

61. Pehlivan E, Turna A, Gurses A, Gurses HN. The effects of preoperative short-term intense physical therapy in lung cancer patients: A randomized controlled trial. Ann Thorac Cardiovasc Surg 2011;17:461-468.

62. Morano MT, Araújo AS, Nascimento FB, et al. Preoperative pulmonary rehabilitation versus chest physical therapy in patients undergoing lung cancer resection: A pilot randomized controlled trial. Arch Phys Med Rehabil 2013;94:53-58.

63. Benzo R, Wigle D, Novotny P, et al. Preoperative pulmonary rehabilitation before lung cancer resection: Results from two randomized studies. Lung Cancer 2011;74:441-445.

64. Bobbio A, Chetta A, Ampollini L, et al. Preoperative pulmonary rehabilitation in patients undergoing lung resection for non-small cell lung cancer. Eur J Cardiothorac Surg 2008;33:95-98.

65. Weinstein H, Bates AT, Spaltro BE, et al. Influence of preoperative exercise capacity on length of stay after thoracic cancer surgery. Ann Thorac Surg 2007;84:197-202.

66. Chesterfield-Thomas G, Goldsmith I. Impact of preoperative pulmonary rehabilitation on the Thoracoscore of patients undergoing lung resection. Interact Cardiovasc Thorac Surg 2016; 23:729-732.

67. Tarumi S, Yokomise H, Gotoh M, et al. Pulmonary rehabilitation during induction chemoradiotherapy for lung cancer improves pulmonary function. J Thorac Cardiovasc Surg 2015;149:569-573.

68. Tokarski S, Tokarska K, Schwarz E, et al. Blood gas analysis, blood saturation and chosen parameters of spirometric examination in NSCLC patients undergoing chemotherapy and pulmonary rehabilitation. Pol Merkur Lekarski 2014;36:249-253.

69. Yankelevitz DF, Yip R, Smith JP, et al. CT screening for lung cancer: Nonsolid nodules in baseline and annual repeat rounds. Radiology 2015;277:555-564.

70. Aberle DR, DeMello S, Berg CD, et al. Results of the two incidence screenings in the National Lung Screening Trial. N Engl J Med 2013; 369:920-931.
71. Siegel R, Naishadham D, Jemal A. Cancer statistics, 2013. CA Cancer J Clin 2013;63:11-30.

72. Howlader N, Noone AM, Krapcho M, et al. SEER stat fact sheets: Lung and bronchus. Bethesda, MD: National Cancer Institute; 2011.

73. Yang P, Cheville AL, Wampfler JA, et al. Quality of life and symptom burden among long-term lung cancer survivors. J Thorac Oncol 2012;7:64-70.

74. Sarna L, Evangelista L, Tashkin D, et al. Impact of respiratory symptoms and pulmonary function on quality of life of long-term survivors of non-small cell lung cancer. Chest J 2004;125: 439-445.

75. Ostroff JS, Krebs P, Coups EJ, et al. Health-related quality of life among early-stage, non-small cell, lung cancer survivors. Lung Cancer 2011;71:103-108.

76. Coups EJ, Park BJ, Feinstein MB, et al. Physical activity among lung cancer survivors: Changes across the cancer trajectory and associations with quality of life. Cancer Epidemiol Biomarkers Prev 2009; 18:664-672.

77. Silver JK, Baima J, Mayer RS. Impairment-driven cancer rehabilitation: An essential component of quality care and survivorship. CA Cancer J Clin 2013;63:295-317.

78. Vijayvergia N, Shah PC, Denlinger CS. Survivorship in non-small cell lung cancer: Challenges faced and steps forward. J Natl Comprehensive Cancer Network 2015;13:1151-1161.

79. Earle CC, Chretien Y, Morris C, et al. Employment among survivors of lung cancer and colorectal cancer. J Clin Oncol 2010;28: 1700-1705.

80. Torp S, Nielsen RA, Fosså SD, et al. Change in employment status of 5-year cancer survivors. Eur J Public Health 2013;23:116-122.

81. Smith SR, Reish AG, Andrews C. Cancer survivorship: A growing role for physiatric care. PM R 2015;7:527-531.

82. Denlinger CS, Ligibel JA, Are M, et al. NCCN clinical practice guidelines insights: survivorship, version 1.2016. J Natl Compr Canc Netw 2016;14:715-724.

83. Chen HM, Tsai CM, Wu YC, et al. Randomised controlled trial on the effectiveness of home-based walking exercise on anxiety, depression and cancer-related symptoms in patients with lung cancer. Br J Cancer 2015;112:438-445.

84. Renfroe KL. Effect of progressive relaxation on dyspnea and state anxiety in patients with chronic obstructive pulmonary disease. Heart Lung 1988;17:408-413.

\section{Disclosure}

S.R.S. Department of Physical Medicine and Rehabilitation, University of Michigan, 325 E Eisenhower Pkwy, Ste 100, Ann Arbor, MI 48108. Address correspondence to: S.R.S.; e-mail: srsz@med.umich.edu

A.K. Division of Rehabilitation Medicine, MedStar National Rehabilitation Hospital, Washington, DC
E.M.W. Division of Rehabilitation Medicine, MedStar National Rehabilitation Hospital, Washington, DC

Submitted for publication February 20, 2017; accepted June 2, 2017. 